\title{
An Experimental Study on Fear of Public Speaking Using a Virtual Environment
}

\author{
Mel Slater ${ }^{1}$, David-Paul Pertaub ${ }^{1}$, Chris Barker ${ }^{2}$, David Clark ${ }^{3}$ \\ 1. Department of Computer Science, University College London, UK \\ 2. Department of Psychology, University College London, UK \\ 3. Institute of Psychiatry, Kings College London, UK \\ Telephone: +44 207679 3709, Fax: +44 2073871397 \\ E-mail:m.slater@cs.ucl.ac.uk
}

\begin{abstract}
This paper examines a necessary condition for successful exploitation of a Virtual Environment (VE) in therapeutic intervention for fear of public speaking. The condition is that clients experience a degree of anxiety in the VE that is similar to what they would have been expected to experience in a similar real world setting. We refer to this as a 'presence' response. The experimental study involved 20 people who were confident public speakers and 16 who were phobic, assessed on a standard psychological scale. Half of each group spoke within a VE depicting an empty seminar room, and the other half within the same room but populated by a neutrally behaving virtual audience of five people. Three responses were measured - a questionnaire-based measure of anxiety, a measure of self-focused attention on somatic responses, and actual heart rate. On all responses the people with phobia showed a significant increase in signs of anxiety when speaking to the virtual audience compared to the empty room, whereas the confident people did not. The result was strong in spite of the relatively low representational and behavioral fidelity of the virtual characters.
\end{abstract}

\section{Keywords}

Social Phobia, Fear of Public Speaking, Virtual Environments, Presence

\section{Introduction}

There have been several reports to date on the exploitation of virtual reality in the context of the treatment of social phobias, for example [2][4][14], as well as plenty of unpublished actual treatment. The premise of such treatment must be what we refer to as the 'presence response': that the degree of anxiety experienced by a client within a virtual reality needs to be sufficiently similar to what they would normally experience in a similar real world situation. Were this not the case then therapeutic intervention may not be successful, or if successful then due to reasons other than the exploitation of virtual reality. The usual demonstration of this premise is that the treatment appears to be successful. In this paper we try to place the presence response on firmer methodological ground, by directly considering people's reaction to a virtual social situation in the context of an experiment. We limit our consideration to one kind of social phobia, fear of public speaking.

40 people were recruited to an experiment on 'public speaking' within a VE. Half of them had public speaking phobia (phobics), and the others were confident public speakers (confidents). Each person was randomly assigned to one of two conditions: they would give a talk within a virtual seminar room populated by seemingly interactive virtual people ('audience' group), or they would give a talk within the same room but which had no 
audience ('empty room'). Each cell of this 2 by 2 table had an equal number of men and women assigned to it. Each person gave a 5 minute talk. Three different response measures were used: a score on a standard public speaking phobia questionnaire, a self-assessment of somatic response, and actual heart-rate measurements. The predicted result from a corresponding real situation was that the confident speakers would exhibit no difference between the two different situations (empty room or audience), whereas the people with public speaking phobia would exhibit markedly different measurable behavior under the two conditions, indicating higher anxiety in response to the virtual audience.

\section{Background}

There has been growing interest in the application of virtual reality to issues in mental health. Almost all examples deal with specific phobias, fear of special situations or objects, such as fear of heights, spiders, open spaces (agoraphobia), and flying. For an overview of the field see [7][12]. There has also been an application to more general syndromes such as post-traumatic stress disorder [13]. Each of these exploits the power of virtual reality to create situations in which a person can be immersed, but under the control of the psychologist, with most therapies involving increasing exposure of the patient to the feared situation.

More computationally challenging are phobias or conditions that involve other people - the social phobias. These are defined as: "A marked and persistent fear of one or more social or performance situations in which the person is exposed to unfamiliar people or to possible scrutiny by others. The individual fears that he or she will act in a way (or show anxiety symptoms) that will be humiliating or embarrassing" [1]. The 'performance situation' of public speaking is the particular social phobia on which we concentrate in this paper. This has been studied experimentally in VEs. North et al. [8] carried out an experiment using a static (texture mapped) large audience that responded in terms of sound only. In earlier work [11] we studied the responses of people to small audiences in a seminar-style setting. Each participant in the experiment experienced either a static audience, an audience that was extremely negative, or an audience that was extremely positive. The purpose was to assess the extent of presence - that is, whether people would respond to these audiences at all, even though everyone involved knew that there was really no audience there. The results showed that the responses of people to the static or positive audience were positively and significantly correlated with their public speaking anxiety measured before their virtual exposure. However, the reported anxiety in response to the negative audience was very high independently of their normal every-day anxiety. This showed that people do respond to these virtual characters in a manner similar to their behavior in real life - that an extremely negative audience can provoke anxiety in almost anyone, no matter how confident.

The earlier study relied exclusively on questionnaires. Moreover, the virtual audiences used were deliberately designed to be unusual in their behavior compared to real life - either overly positive (finishing with a standing ovation), or very negative (the audience members turning away from the speaker and even walking out during the talk). Perhaps a VE depicting an extreme social situation evokes a response simply because of the extremity itself. Although our earlier study provided some evidence in favor of the notion that people become present in virtual social situations despite the relatively poor representational and behavioral fidelity, further evidence is required, hence this additional study.

In the present experiment we use one virtual audience throughout - a neutral dynamic audience with no obvious 'positive' or 'negative' behaviors towards the speakers. Instead of varying the types of behavior of the virtual characters we have different types of participants (phobics or confidents) whose responses should be different for the same audience (if there is presence). Crucially our experimental design addresses the question as to whether the anxiety that is experienced is due simply to the fact of being immersed in a virtual reality or whether the virtual audience itself makes a difference.

\section{The Experiment}

\subsection{Recruitment}

The experimental participants were recruited by advertisements in local newspapers and throughout the university campus. On first contact they were asked to complete a standard questionnaire that assesses degree of public speaking anxiety (PRCS: Personal Report of Confidence as a Public Speaker [10]). This consists of 30 
statements each of which the participant agrees with or not ('yes' or 'no'). For example, five of these statements are:-

- $\quad$ I am in constant fear of forgetting my speech.

- $\quad$ At the conclusion of a speech I feel that I have had a pleasant experience.

- $\quad$ My thoughts become confused and jumbled when I speak before an audience.

- $\quad$ I have no fear of facing an audience.

- $\quad$ - Although I am nervous just before getting up, I soon forget my fears and enjoy the experience.

The PRCS is scored by counting the number of answers indicating anxiety. Of all the people who responded to the advertisement, those who had a score in the bottom third of the range (10 or less) were assigned to the confident group, those with a score in the top third of the range (20 or more) were assigned to the phobic group, and others were not included in the study. Subjects were also not included in the experiment if there was evidence of psychotism. This process of participant selection continued until 41 people had been recruited (the extra one to allow for drop-outs). The experiment was approved by the University Ethics Committee.

\subsection{Scenario}

There were two scenarios. The first was a seminar room with a table and chairs. The second used the same room but populated with an audience of five animated virtual humans (avatars) two women and three men dressed in casual style seated around the table (Figure 1). The responses of the audience did not depend upon the content of the speech being given. A range of neutral audience behavior was developed, focusing in particular on:

- The movement of the upper face and subtle cues that indicate the degree of interest the audience member is paying, such as transient frowns or furrowed brows.

- $\quad$ Eye contact and direction of gaze (Figure 1).

- Gestures with no intrinsic evaluative content, such as self grooming, and those whose meaning is ambiguous, such as taking out a pen, looking at notes etc. (Figure 2).

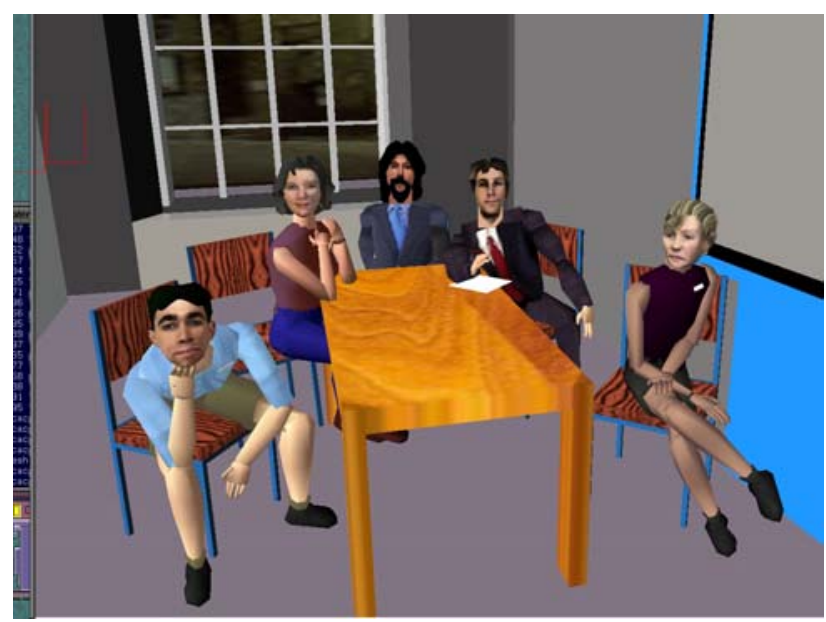

Figure 1: Facial Expressions and Gaze Direction

\section{3. $\quad$ Procedures}

Participants attended the Department at pre-arranged times, and were provided with an information sheet, and given an informed consent sheet to sign if they agreed to take part in the study. Participants were fitted with a Polar chest strap monitor recording heart rate and then given about 5 minutes to prepare a short talk on a subject of their choice (a list of possible topics was given), to be delivered without notes or other visual aids.

Participants donned a head-mounted display in the virtual reality laboratory, and gave their talk to the virtual audience. During the talk, their heart rate was monitored and the content of the presentation was recorded. An 
experimenter was in a remote location, unseen by the participants, watching the display and listening. After the presentation was finished, participants completed a further questionnaire designed to explore their subjective experience of the virtual environment. Afterwards there was a debriefing session, when they were given the opportunity to expand upon their experience, and the purpose of the experiment explained to them. They were asked not to discuss the experiment with anyone else for a period of one month. They were paid the equivalent of $\$ 9$.

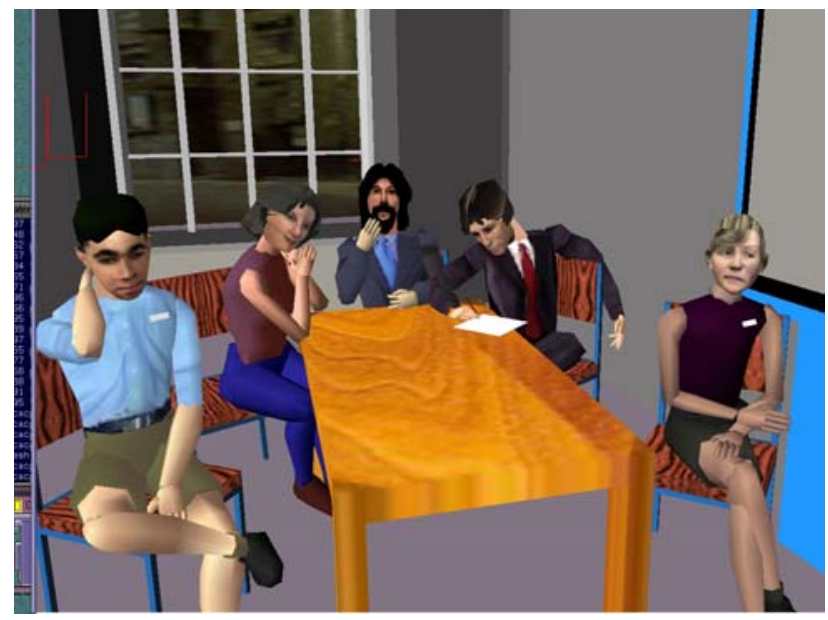

Figure 2: Self Grooming and Ambiguous Behaviors

\subsection{Equipment and Software}

The virtual reality public speaking scenarios were implemented using DIVE (http://www.sics.se/dive/). The avatar faces and accompanying muscle model used Parke and Waters [9] and were texture mapped to provide the different faces. Clothing was texture mapped onto each avatar. The experiments were conducted on a Silicon Graphics Onyx with twin 196 Mhz R10000, infinite reality graphics and 192MB main memory. The headtracking system was with a Polyhemus Fastrak. The helmet was a Virtual research VR8.

\subsection{Removal of Outliers}

Originally there were 20 participants in each group (phobic, confident) who were randomly allocated to one of two conditions - to talk to the empty virtual room or to the audience. In spite of the random allocation, outliers were indicated by the mean PRCS scores for the phobic group. The phobic group who spoke to the empty room had mean and standard deviation PRCS score 23.0 2.5 , whereas those who spoke to the audience had mean score 26.4 \pm 3.4 . This difference, noticed after completion of the experiment, is significant at the $5 \%$ level. For the experimental results to be valid, these PRCS means, measured prior to the experiment, should not be significantly different. In order to equalize the two groups with respect to PRCS, participants were deleted from the data as follows: the individuals with the highest PRCS in the phobic-audience group and with the lowest in the phobic-empty group were removed, and the means tested for significant difference. This was repeated twice, and on the second pair of removals the means were no longer significantly different $(\mathrm{P}=0.18)$. Gender balance was maintained. The mean for the empty room became $23.6 \pm 2.4$, and for the audience group $25.7 \pm 3.5$, each now based on 8 observations. This reduced data set is used throughout.

The procedure for removing outliers at worst can reduce the power of the subsequent statistical tests, in other words reduce the probability of such tests differentiating between the 'empty' and 'audience' conditions even if the underlying populations would have different mean responses. A priori we would expect PRCS measured before the experiment to be correlated with the anxiety response measured after the experiment. By equalizing prior-experiment PRCS we are making it less likely that there will be a difference in post-experiment anxiety between these two groups. 


\section{Results}

\subsection{Post Talk PRCS}

A modified PRCS was used as a response variable to assess the degree of anxiety related specifically to the talk (rather than to general everyday experience). This was constructed by modifying the standard PRCS to refer to the talk just given, hence changing some of the tenses, and removing some questions that were inappropriate because they refer only to the general situation. For example, the 5 questions mentioned in Section 2 became:

- I was in constant fear of forgetting my speech.

- $\quad$ At the conclusion of the speech I felt I'd had a pleasant experience.

- $\quad$ My thoughts became confused and jumbled when I spoke.

- I had no fear of facing the audience.

- Although I might have felt nervous before I began to speak, I soon forgot my fears and enjoyed the experience.

After deleting statements that were not relevant 15 questions were left, referred to as the post-talk PRCS (PTPRCS), indicating the anxiety experienced due to the talk just given in the VE. Our expectation was that the presence response would result in a greater anxiety for the phobic group when talking to the audience compared to talking to the empty room, but that there would be no difference between these two situations for the confident group. Of course we would expect much greater PT-PRCS for the phobic compared to the confident group. The result is shown in Table 1 . The difference in means for the phobic group is highly significant (using a two-tailed t-test, $\mathrm{P}=0.013)$ but the means for the confident group are not significantly different $(\mathrm{P}=0.8)$. Of course there is a highly significant difference between the means for the phobic and confident groups $(\mathrm{P}=0.01$ for the empty room, and $\mathrm{P}=6.0^{\prime} 10-7$ for the audience).

Table 1 PT-PRCS Mean+SD

\begin{tabular}{|l|c|c|}
\hline & empty & audience \\
\hline phobic & $6.50 \pm 3.34$ & $10.38 \pm 1.30$ \\
\hline confident & $2.40 \pm 2.76$ & $2.70 \pm 2.45$ \\
\hline
\end{tabular}

Table 2 Somatization Mean \pm SD

\begin{tabular}{|l|c|c|}
\hline & empty & audience \\
\hline phobic & $88.9 \pm 43.1$ & $158.8 \pm 37.2$ \\
\hline confident & $42.2 \pm 39.1$ & $42.2 \pm 39.1$ \\
\hline
\end{tabular}

In Pertaub et al. [11] we used a more appropriate method of analysis for the PT-PRCS, which is to recognize that this response is a count variable (the number of anxiety statements made out of 15) rather than a continuous normal variate, and therefore we used binomial logistic regression (equivalent to logistic two-way analysis of covariance) instead. When this is done the same conclusions are reached. That approach also allowed us to add in the original (pre-experiment) PRCS scores as a covariate, to further eliminate the impact of this variable. When this additional term is added into the regression model the results remain significant, and the same conclusions are reached.

\subsection{Somatization}

An important component of the behavior of people with a social phobia is their inward focus on their bodily processes and their belief that others are also aware of these. This is often referred to as 'self-focused attention' 
[3]. For example, a slight increase in heart rate might be subjectively experienced as a massive increase, and sufferers will also tend to believe that onlookers will be aware of this condition. The APQ questionnaire [8] assesses the extent of such somatization by presenting participants with a series of statements and asking them to assess on a scale their appropriateness as a description of their own subjective state. For example, these included: Awareness of many bodily sensations, trembling or shaking, dizziness, face becoming hot, perspiration, and so on. Participants were asked to draw a cross at the point on each scale corresponding to their experience, and also separately assess their belief about how others would rate them. These two sets of scores are combined into an overall scale, such that the higher the score the greater the degree of subjective somatization.

The results are shown in Table 2. Clearly there is a large significant difference for the phobic group $(\mathrm{P}=0.004)$ and no difference for the confident group. (When PRCS is used as a co-variate using the normal analysis of covariance model, there is no change in these results).

\subsection{Heart Rate}

Heart rate has been used before in the study of presence. It was used in [6] in the context of a stressful VE. In the current experiment heart rate was measured for all participants during their talks and also during a resting period afterwards. Readings were available for 5 minutes during the talk period, and for 5 minutes after the talk. In each case only the last 4 minutes of recordings were used, in order to allow one minute for a settling down period. Heart rate was sampled at 5 second intervals as a moving average. Hence there were 48 observations over each of the two 4-minute periods of recording (during the talk and also for the resting period afterwards). Each person's heart rate was standardized by subtracting their mean for the whole period, then the individual series were averaged over the participants to produce a sequence for each experimental condition. This standardization implies an overall zero mean for each sequence.

Of critical importance are the differences between trends of the time-series for the phobics under the two conditions, and also between the phobics and confident speakers under the audience condition. Also of interest, as a control, is to check that the resting heart rate series do not differ significantly between the various groups in which case we can be sure that the heart rates are being affected by the presence of the virtual audience, and nothing else. Figure 3 shows a time-series plot for the two groups of phobics. It is clear that there is a quite different pattern for those who spoke to the audience compared to those who spoke in the empty room. (Recall that the series are standardized to have zero mean).

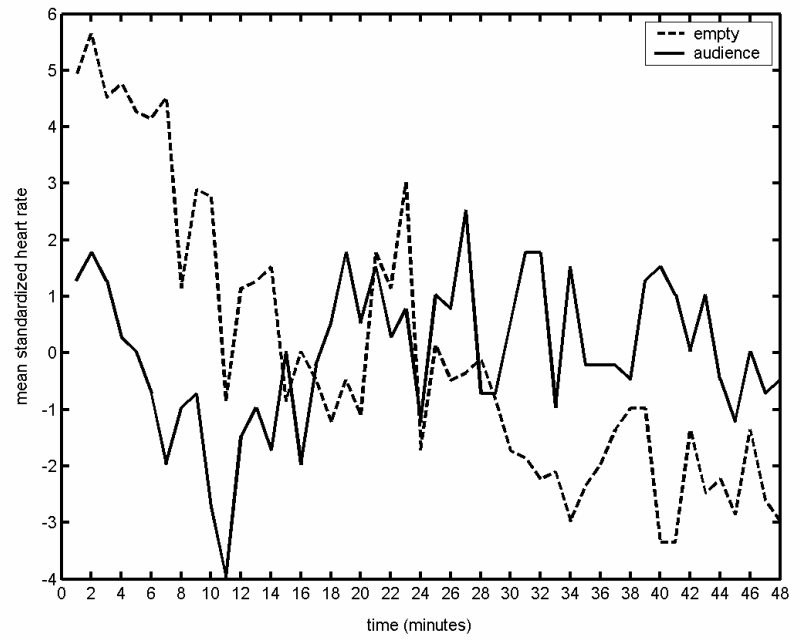

Figure 3: Mean Standardized Heart Rate - Phobics during talk. Dashed line: empty room, solid : audience.

The ith cross correlation between two time series $x(t)$ and $y(t)$ is the correlation between time shifted series $x(t)$ and $y(t+i)(i=0, \pm 1, \pm 2, \pm 3, \ldots)$. Of particular interest are windows around $i=0$, which show the correlations of the original series, slightly time-shifted to allow for different starting points for the underlying trends.

A cross correlation analysis for the mean standardized heart rates for the phobics (Figure 4, solid) shows that vast majority of the correlations between time-shifted versions of the two series are negative and significant for approximately $-20<i<20$ (note that $|r|>0.284$ is significant at the $5 \%$ level on series of length 48). The same 
Figure shows the cross correlations between the confidents who spoke to the empty room, and those who spoke to the audience (dashed). In this case almost all the correlations are within the non-significantly different from zero bounds.

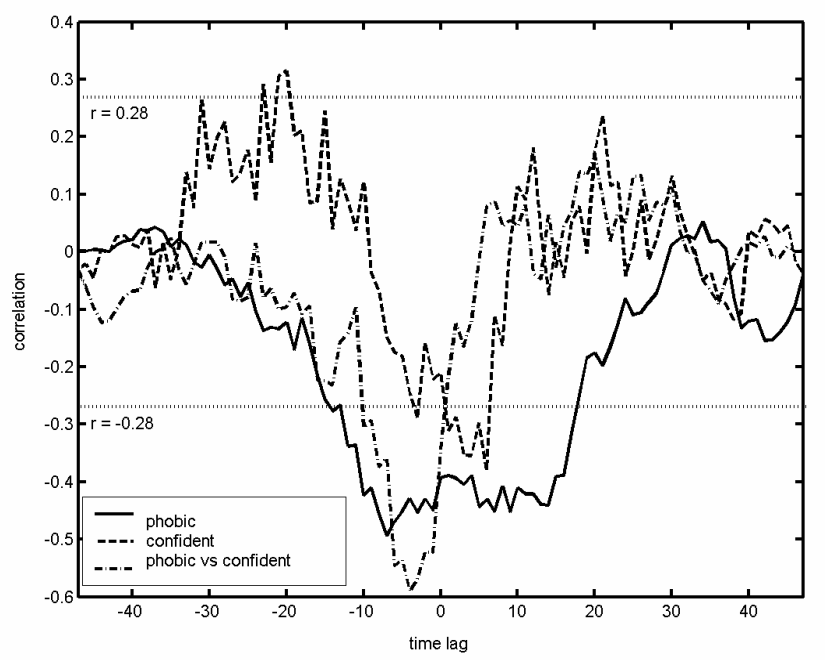

Figure 4: Cross Correlations for the phobics (audience and empty room - solid line), confidents (audience and empty room, dashed), and between phobics and confidents who spoke to the audience, dash-dot).

Comparing now the phobic and confident groups who spoke to the audience (Figure 5) we see again a clear difference between their responses (the series are almost reflections of each other about $y=0$ ). The crosscorrelation analysis also shows strong overall negative correlations between the two series (Figure 4, dash-dot).

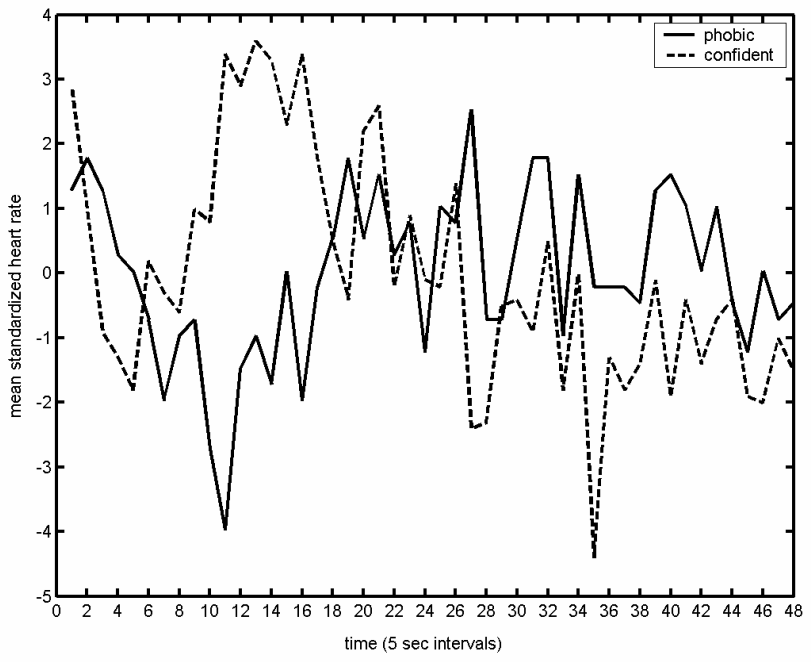

Figure 5: Mean Standardized Heart Rate - Phobic and Confident Speakers with the Audience.

Finally we consider the resting heart rate series. In this case we are looking for zero or positive correlations between the resting heart rates amongst the two phobic groups (those who had spoken to the empty room and those who had spoken to the audience) and similarly for the confident groups. Figure 6 shows both of the crosscorrelation plots. The correlations are not significantly different from zero or are positive, in both cases the peak correlation is significant and positive. This is the opposite pattern from the equivalent cross-correlations based on heart rate data during the talks. In other words, during the resting period after the experience, the heart rate series are similar for both phobic groups, and also for both confident groups. 


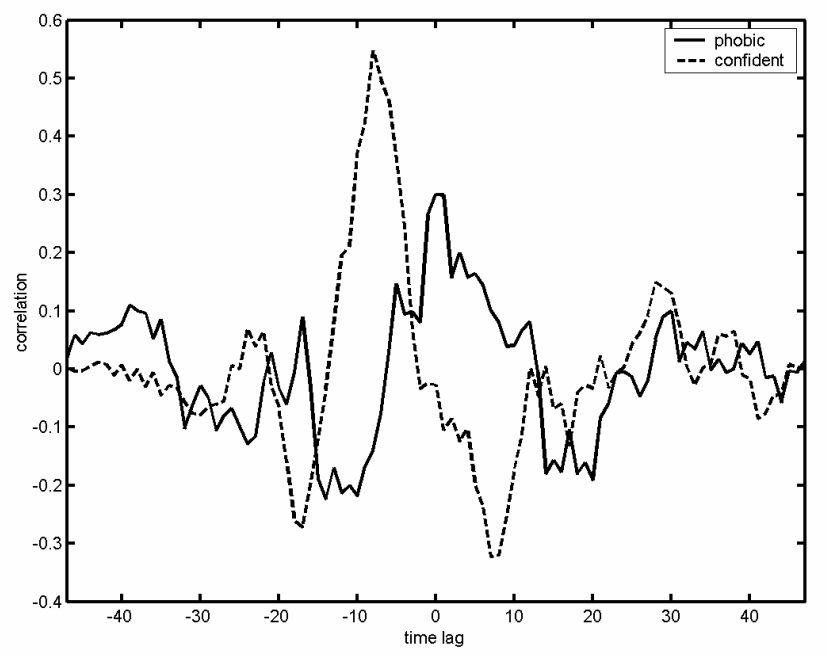

Figure 6 : Cross-Correlation for the two Phobic Groups and for the two Confident Groups during the post-talk Rest Period.

\section{Conclusions}

This research has shown that a strong presence response can be obtained with a relatively low fidelity VE depicting a social encounter (in this case, a person giving a talk). In spite of the fact that the avatars had low representational quality, with pre-programmed behaviors independent of the actual behavior of the speaker, people still responded according to type. Confident speakers did not have a different response between speaking to an empty room and speaking to an animated audience. The groups of phobic speakers exhibited differences in anxiety in three ways: those speaking to the audience reported questionnaire-based anxiety that was significantly higher than those speaking to the empty room, and their subjective assessment of their somatic response was almost doubled. Those who spoke to the empty room had a decreasing trend in heart rate compared to those who spoke to the audience. The phobics who spoke to the audience exhibited heart rate trends significantly different from the confident speakers who spoke to the audience. As a control, there were no relevant differences in the heart rate responses during the resting period after the experiment. It is emphasized that this was a betweengroups study, i.e., each individual experienced only one condition, so that there was no possibility of a learning effect between conditions.

Previous studies have used unusual environments to generate an anxiety response [11][6]. Here we have shown that the anxiety response occurs for a relatively neutral situation, but varies appropriately for different types of people - those with phobic tendencies exhibit anxiety, and those without such tendencies do not. This is what would be expected in similar circumstances in everyday reality, thus indicating a presence response in the VE.

The behaviors of the avatars were designed based on careful observations of people in real meetings. An important question that needs answering is how much lower could the representational and behavioral fidelity become while maintaining the same results, and conversely how much would be gained by investing resources in order to significantly improve such fidelity? In any case the results suggest that the necessary condition for appropriate anxiety to be generated within the VE appears to be satisfied, though, of course, this condition may not be sufficient for successful treatment. In order to further answer this question, to date we have carried out three individual case studies based on this scenario, that will be reported in a later paper.

\section{Acknowledgements}

This research was supported by the Wellcome Foundation, and also by the UK EPSRC Equator IRC. Thanks to Anthony Steed for helping with DIVE and to Amanda Slater for helping with the experimental sessions. 


\section{References}

1. American Psychiatric Association. Diagnostic and Statistical Manual of Mental Disorders, Fourth Edition (DSM-IVTM), Primary Care Version, American Psychiatric Association, ISBN 0-89042- p.p. 407-1, 1995.

2. Anderson, P., Rothbaum, B. O., \& Hodges, L. F. Virtual reality in the treatment of social anxiety: Two case reports. Cognitive and Behavioral Practice, 10, p.p. 240-247, 2003.

3. Clark, D.M., And Wells, A. A cognitive model of social phobia. In R.G. Heimberg, M.R. Liebowitz, D.R. Hope, and F.R. Schneier (Eds.) Social phobia: Diagnosis, assessment and treatment. New York: Guilford, 1995.

4. Harris S.R., Kemmerling R.L., North M.M. Brief Virtual Reality Therapy for Public Speaking Anxiety, CyberPsychology \& Behavior, 5(6), p.p. 543-550, 2002.

5. Mandler, G.., Mandler, J.M., and Uviller, E.T. Autonomic feedback: The perception of autonomic activity, Journal of Abnormal and Social Psychology, 56, p.p. 367-373, 1958.

6. Meehan, M., Insko, B., Whitton, M., Brooks, F.P. Physiological Measures of Presence in Stressful Virtual Environments, ACM Transactions on Graphics, 21,3, p.p. 645-653, 2002.

7. Molinari, E., Riva, G. and Wiederhold, B.K. (eds.). Virtual Environments in Clinical Psychology and Neuroscience, IOS Press, 1998.

8. North, M.M., North S.M. And Coble, J.R. Virtual Reality Therapy: An effective treatment for the fear of public speaking, International Journal of Virtual Reality 3,2, p.p. 2-6, 1998.

9. $\quad$ Parke, F. And Waters, K. Computer Facial Animation, A.K.Peters, 1998.

10. Paul, G. Insight vs Densensitisation in Psychotherapy, Stanford University Press, 1966.

11. Pertaub, D-P., Slater, M., Barker, C. An Experiment on Public Speaking Anxiety in Response to Three Different Types of Virtual Audience, Presence: Teleoperators and Virtual Environments, 11,1 p.p. 68-78, 2001.

12. Rizzo, A.A., Buckwalter, J.G. \& van der Zaag, C. Virtual Environment Applications for Neuropsychological Assessment and Rehabilitation. In Stanney, K. (Ed.) Handbook of Virtual Environments. L.A. Earlbaum: New York. p.p. 1027-1064, 2002.

13. Rothbaum, B.O., Hodges, L.F., Alarcon, R., Ready, D., Shahar, F., Graap, K., Pair, J., Herbert, P., Gotz, D., Wills, B. and Baltzell, D. Virtual Reality Exposure Therapy for PTSD Vietnam Veterans: A Case Study. Journal of Traumatic Stress 12,2, p.p. 263-271, 1999.

14. Roy, S., Klinger, E., Légeron, P., Lauer, F., Chemin, I., Nugues, P. Definition of a VR-Based Protocol to Treat Social Phobia, CyberPsychology \& Behavior, 6(4), p.p. 411-420, 2003. 